Trinity University

Digital Commons@ Trinity

Chemistry Faculty Research

Chemistry Department

3-18-2015

\title{
Sequence-Specific, Nanomolar Peptide Binding via Cucurbit [8] uril-Induced Folding and Inclusion of Neighboring Side Chains
}

Lauren C. Smith

Trinity University, 1smith16@trinity.edu

David G. Leach

TrinityUniversity, dleach@trinity.edu

Brittney E. Blaylock

TrinityUniversity, bblayloc@trinity.edu

Omar A. Ali

TrinityUniversity, oali@trinity.edu

Adam R. Urbach

Trinity University, aurbach@trinity.edu

Follow this and additional works at: https://digitalcommons.trinity.edu/chem_faculty

Part of the Chemistry Commons

\section{Repository Citation}

Smith, L. C., Leach, D. G., Blaylock, B. E., Ali, O. A., \& Urbach, A. R. (2015). Sequence-specific, nanomolar peptide binding via cucurbit[8] uril-induced folding and inclusion of neighboring side chains. Journal of the American Chemical Society, 137(10), 3663-3669. doi: 10.1021/jacs.5b00718

This Post-Print is brought to you for free and open access by the Chemistry Department at Digital Commons @ Trinity. It has been accepted for inclusion in Chemistry Faculty Research by an authorized administrator of Digital Commons @ Trinity. For more information, please contact jcostanz@trinity.edu. 


\title{
Sequence-Specific, Nanomolar Peptide Binding via Cucurbit[8]uril- Induced Folding and Inclusion of Neighboring Side Chains
}

\author{
Lauren C. Smith, David G. Leach, Brittney E. Blaylock, Omar A. Ali, Adam R. Urbach* \\ Department of Chemistry, Trinity University, San Antonio, TX 78212
}

\begin{abstract}
This paper describes the molecular recognition of the tripeptide Tyr-Leu-Ala by the synthetic receptor cucurbit[8]uril (Q8) in aqueous buffer, with nanomolar affinity, and with exceptional specificity. This combination of characteristics, which also applies to antibodies, is desirable for applications in biochemistry and biotechnology but has eluded supramolecular chemists for decades. Building on prior knowledge that Q8 binds to peptides with N-terminal aromatic residues, a library screen of 105 peptides was designed to test the effects of residues adjacent to N-terminal Trp, Phe, or Tyr. The screen used tetramethylbenzobis(imidazolium) (MBBI) as a fluorescent indicator and resulted in the unexpected discovery that MBBI can serve not only as a turn-off sensor via the simultaneous inclusion of a Trp residue, but also as a turn-on sensor via the competitive displacement of MBBI upon binding of Phe- or Tyr-terminated peptides. The unusual fluorescence response of the Tyr series prompted further investigation by ${ }^{1} \mathrm{H}$ NMR spectroscopy, electrospray ionization mass spectrometry, and isothermal titration calorimetry. From these studies, a novel binding motif was discovered in which only one equivalent of peptide binds to Q8, and the sidechains of both the N-terminal Tyr residue and its immediate neighbor bind within the Q8 cavity. For the peptide Tyr-Leu-Ala, the equilibrium dissociation constant value is $7.2 \mathrm{nM}$, whereas that of its sequence isomer Tyr-Ala-Leu is $34 \mu \mathrm{M}$. The high stability, recyclability, and low cost of Q8 combined with the straightforward incorporation of Tyr-Leu-Ala into recombinant proteins should make this system attractive for the development of biological applications.
\end{abstract}

\section{INTRODUCTION}

The discovery and development of synthetic compounds that can recognize specific peptides and proteins underpins the effort to interface with living systems. One of the principal technological successes in this area includes monoclonal antibodies, which bind their peptide targets with equilibrium dissociation constant $\left(\mathrm{K}_{\mathrm{d}}\right)$ values in the nanomolar range and with exquisite specificity. ${ }^{1}$ Supramolecular chemists have developed a wide range of synthetic receptors for peptides in aqueous solution, ${ }^{2-9}$ but most have $K_{d}$ values in the micromolar to millimolar range. Compared to antibodies, however, synthetic receptors offer the advantages of being more economical to produce, more stable, more scalable, and more reliable. These advantages, however, will only become relevant for biological applications if the receptor can recognize a peptide in aqueous solution, at nanomolar concentrations, and with at least 1000-fold sequence specificity, as described here for the synthetic receptor cucurbit[8]uril (Q8).

Q8 (Figure 1) derives from the cucurbit[n] uril (Qn) family of organic macrocycles, ${ }^{10}$ which have earned distinction for their capacity to encapsulate a wide array of organic molecules in primarily aqueous media with $K_{d}$ values that can extend to attomolar levels. ${ }^{11-13}$ Numerous Qn homologues, ${ }^{14,15}$ derivatives, ${ }^{16-20}$ and acyclic congeners $^{21}$ have extended the family to myriad structures and applications. $^{22}$ Q8 and its homologue cucurbit[7]uril (Q7) have been shown to bind amino acids, peptides, and proteins, with preference for the aromatic residues tryptophan (Trp), phenylalanine (Phe), and tyrosine (Tyr), especially when located at the N-terminal position in the polypeptide chain. ${ }^{6}$ An Nterminal aromatic residue is recognized in the same fashion as many other Qn-based guests, which is by inclusion of the hydrophobic group (the side chain) within the nonpolar cavity and stabilization of the cationic group (the N-terminal ammonium) with the carbonyl oxygens that surround the two constricted entrances to the Qn cavity. Q8 is large enough to include two aromatic guests within its cavity, ${ }^{23,24}$ and the earliest report of peptide recognition by the Qn family ${ }^{25}$ involved a ternary complex of Q8 with methyl viologen (MV) and the indole sidechain of a Trp-containing peptide. In this system, Q8 binds 
first to MV, and the Q8 $\bullet \mathrm{MV}$ complex then binds to the indole side chain of a Trp-containing peptide. Specificity for Trp at the N-terminal position is gained by interaction with the N-terminal ammonium group when it is part of the Trp residue and thus proximal to the indole side chain.

In order to explore the scope and limitations of affinity and selectivity in the Q8 $\bullet \mathrm{MV} \bullet \operatorname{Trp}$ system, we studied the effects of residues adjacent to Trp and found little, if any, influence on binding to $\mathrm{Q} 8 \cdot \mathrm{MV} .^{26}$ That study, however, was limited to Trp-containing peptides due to the need for a fluorophore. We have shown that MV can be replaced by tetramethyl benzobis(imidazolium) (MBBI) while still maintaining the molecular recognition properties for Trp-containing peptides. ${ }^{27}$ The intrinsic fluorescence of MBBI broadens the scope of possible analytes to include nonfluorescent guests, thus enabling the exploration of a larger peptide sequence space, the first report of which is presented here.

\section{RESULTS AND DISCUSSION}

Design and screen of a peptide library. A peptide library (Figure 1) was designed to examine the effects of amino acid sequence on the binding of Q8 to peptides containing an aromatic residue at the Nterminus. All compounds were tripeptides comprising natural, unmodified side chains. The N-terminal residue was Trp, Phe, or Tyr. The residue immediately adjacent to the N-terminal residue ( $\operatorname{Var}_{1}$ ) or two residues away ( $\operatorname{Var}_{2}$ ) was varied among 18 genetically encoded amino acids while holding the other position in the tripeptide constant with an alanine (Ala) residue. Trp and cysteine (Cys) were not included at the variable positions due to the complications introduced by having a second Trp binding site and to the fact that Cys residues form disulfide bonds under ambient conditions. This design yielded a library of 105 peptides (leaving out replicates when $\operatorname{Var}_{1}=\operatorname{Var}_{2}=$ Ala). The library was synthesized by parallel solid-phase synthesis on Rink amide Lantern resins, and thus all C-termini were primary amides.

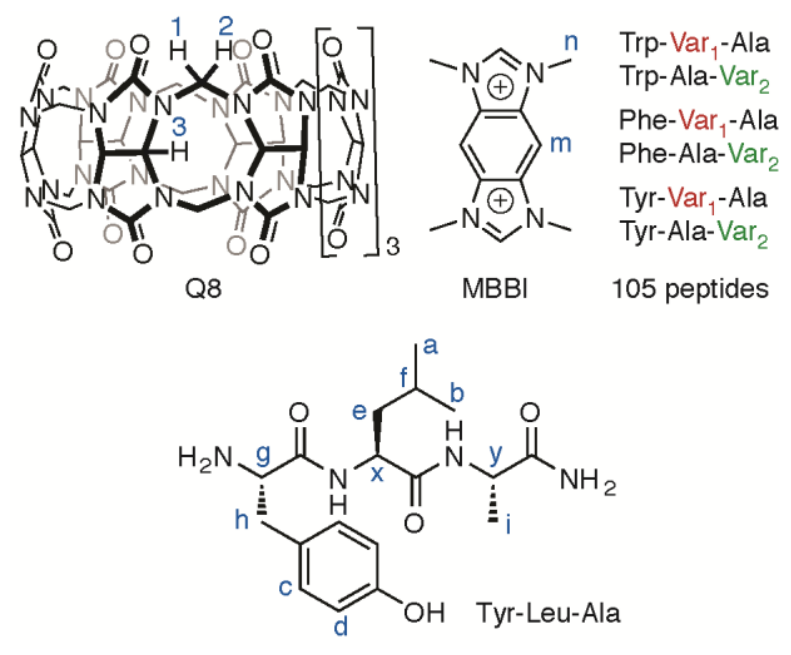

Figure 1. Chemical formulas and peptides sequences used in this study.

Central to the assay design is the fluorophore $\mathrm{MBBI},{ }^{27}$ which was shown previously to have molecular recognition properties similar to MV but with the advantages of intrinsic fluorescence, higher chemical and thermal stability, and greater synthetic tunability. Q8 binds to MBBI with a $K_{d}$ value of 
1.2 $\mu \mathrm{M}$, and the $\mathrm{Q} 8 \cdot \mathrm{MBBI}$ complex binds to Trp-containing peptides with affinities and sequence specificities that are remarkably similar to those of the Q8 $\bullet \mathrm{MV}$ complex. The simultaneous binding of MBBI and a second guest within the Q8 cavity quenches the intrinsic fluorescence of MBBI, and thus the Q8 $\bullet \mathrm{MBBI}$ complex can be used to sense the binding of both fluorescent and non-fluorescent guests.

The peptide library was screened for binding to Q8・MBBI by measuring the change in the fluorescence intensity of Q8•MBBI upon the addition of each peptide in $10 \mathrm{mM}$ sodium phosphate solution, $\mathrm{pH} 7.0$, at room temp. A comparison of the 35 peptides containing an N-terminal Trp residue (Figure S52) revealed that the fluorescence intensity of Q8 $\bullet \mathrm{MBBI}$ generally decreased upon binding to the Trp-containing peptides, as expected. ${ }^{27}$ Compared to Q8•MBBI, fluorescence intensities decreased by $4 \%$ to $57 \%$, and in the singular case of Trp-Phe-Ala, the intensity increased by $4 \%$. The decrease in fluorescence intensity indicates the simultaneous inclusion of MBBI and the indole side chain of Trp within the cavity of Q8, as observed previously. ${ }^{27}$ For the 35 peptides containing an N-terminal Phe residue (Figure S51), fluorescence increased for all peptides, covering the range $0.45 \%$ to $24 \%$. This unexpected increase may be explained in light of two previously reported observations: 1) the fluorescence intensity of free MBBI is higher than that of the Q8•MBBI complex; ${ }^{27}$ and 2) peptides containing an N-terminal Phe residue are known to bind to Q8 in a 2:1 peptide:Q8 stoichiometry. ${ }^{28}$ With these observations in mind, we hypothesized that the increase in fluorescence intensity is due to displacement of MBBI by the peptide.

The most curious result of this experiment, however, involved the 35 peptides with an N-terminal Tyr residue (Figure 2). In this series, the influence of peptide on the observed fluorescence varied significantly and was highly sequence-dependent. Fluorescence intensity decreased for five peptides $(0.21 \%$ to $6.9 \%)$ and increased for 13 peptides $(0.39 \%$ to $21 \%)$. In several cases (Var $=$ Leu, Phe, Tyr, Met, Thr, Ser, and Asn), the fluorescence intensity increased for the peptide Tyr-Var 1 -Ala but decreased

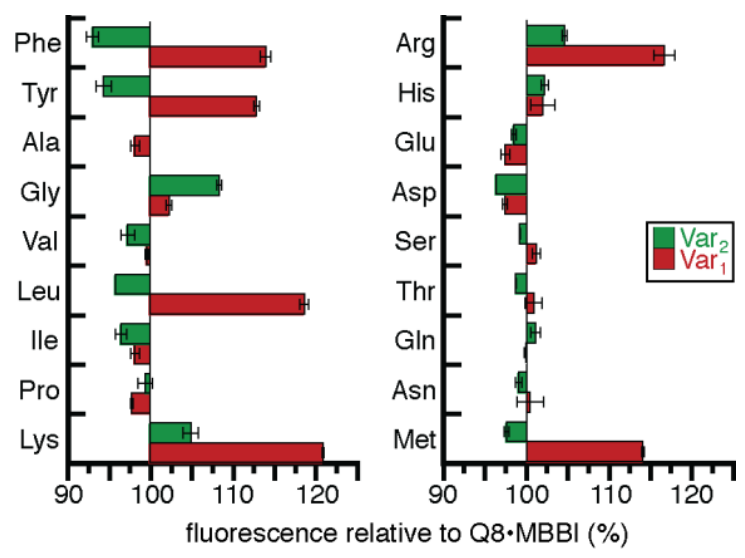

Figure 2. Fluorescence relative to the Q8 $\bullet \mathrm{MBBI}$ complex for mixtures of Q8•MBBI with each of 35 tripeptides of sequence Tyr-Var - -Ala and Tyr-Ala-Var. Samples contained $200 \mu \mathrm{M}$ peptide and $40 \mu \mathrm{M}$ Q8・MBBI at room temp in $10 \mathrm{mM}$ sodium phosphate, $\mathrm{pH}$ 7.0. Values are averages of at least three experiments. Error bars are indicated as the standard deviation.

for the peptide Tyr-Ala-Var2. This remarkable result suggests that there may be a sequence-dependent change in binding mode for these peptides. The largest observed difference (23\%) was observed for Var = Leu (i.e., Tyr-Leu-Ala versus Tyr-Ala-Leu). These two sequence isomers were therefore chosen for in-depth study to further explore this phenomenon. 
In-Depth Study of Tyr-Leu-Ala and Tyr-Ala-Leu. In the library screen described above, the peptides were synthesized in parallel on $8 \mu \mathrm{mol}$ scale and used without further purification (see Supporting Information for experimental details). For the in-depth studies described here, Tyr-Leu-Ala and Tyr-Ala-Leu were synthesized individually on $37 \mu \mathrm{mol}$ scale, purified by reversed phase HPLC, and characterized by ${ }^{1} \mathrm{H}$ NMR and electrospray ionization mass spectrometry (ESI-MS) (Figures S1, S3, $\mathrm{S} 19$, and S25). The library screen showed that the fluorescence intensity of Q8•MBBI increased in the presence of Tyr-Leu-Ala but decreased in the presence of Tyr-Ala-Leu. In that experiment, however, the emission intensity data were collected at only a single wavelength $(340 \mathrm{~nm})$. In order to probe any additional spectral effects, full emission spectra were acquired for Q8•MBBI in the absence and in the presence of Tyr-Leu-Ala and Tyr-Ala-Leu in $10 \mathrm{mM}$ sodium phosphate, $\mathrm{pH} 7.0$ at room temp (Figure 3). Interestingly, the fluorescence spectrum of the mixture containing Q8, MBBI and Tyr-Leu-Ala is essentially identical to that of free MBBI (also shown). This result is consistent with the displacement of MBBI from the cavity of Q8 upon binding of Tyr-Leu-Ala. The fluorescence spectrum of the mixture containing Q8, MBBI, and Tyr-Ala-Leu, however, shows a decrease in fluorescence intensity as compared to that of Q8•MBBI, which is consistent with prior work showing the quenching of Q8•MBBI fluorescence upon the inclusion of the side-chain of Trp. Therefore, we hypothesized that a portion of Tyr-Ala-Leu was bound within the cavity of the Q8•MBBI complex, and further study was undertaken to explore the mode of binding in more detail.

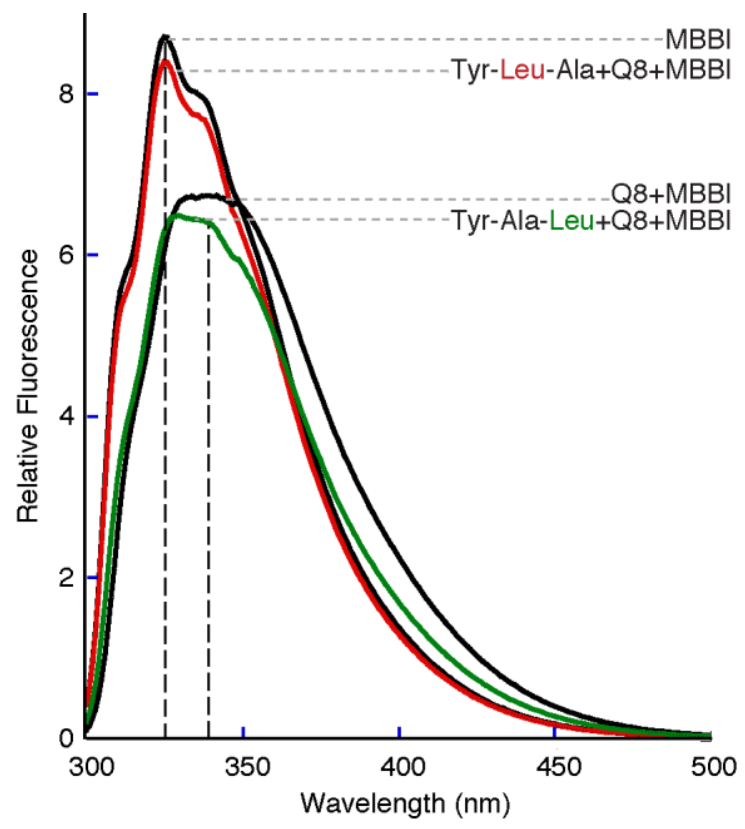

Figure 3. Fluorescence emission spectra $\left(\lambda_{\mathrm{ex}}=297 \mathrm{~nm}\right)$ acquired at room temp for mixtures containing $40 \mu \mathrm{M} \mathrm{MBBI}, 40 \mu \mathrm{M}$ Q8•MBBI, and $40 \mu \mathrm{M}$ Q8•MBBI in the presence of $200 \mu \mathrm{M}$ peptide, all in $10 \mathrm{mM}$ sodium phosphate, $\mathrm{pH}$ 7.0. 


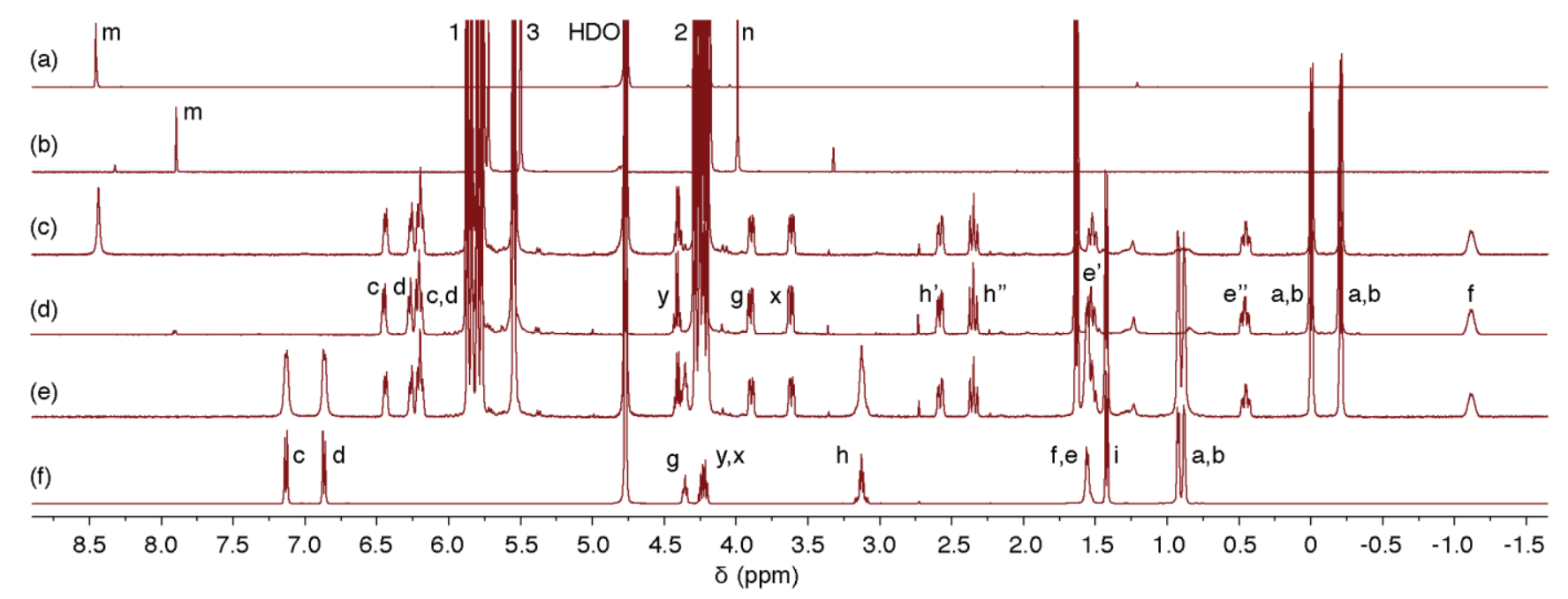

Figure 4. $500 \mathrm{MHz}{ }^{1} \mathrm{H}$ NMR spectra acquired at $25^{\circ} \mathrm{C}$ for samples containing (a) MBBI, (b) a 1:1 mixture of Q8 and MBBI, (c) a 1:1:1 mixture of Q8, MBBI, and Tyr-Leu-Ala, (d) a 1:1 mixture of Q8 and Tyr-Leu-Ala, (e) a 1:2 mixture of Q8 and Tyr-Leu-Ala, and (f) Tyr-Leu-Ala. Samples were approximately $0.5 \mathrm{mM}$ in unbuffered deuterium oxide.

A detailed investigation of the binding of Tyr-terminated peptides was carried out using ${ }^{1} \mathrm{H}$ NMR spectroscopy in deuterium oxide at $25^{\circ} \mathrm{C}$. Binding of a guest or a portion of a guest within the cavity of a cucurbit[n]uril is known to induce an upfield perturbation of the chemical shift of the corresponding signals. ${ }^{29}$ As shown previously, ${ }^{27}$ and again here (Figure $4 \mathrm{a}, \mathrm{b}$ ), this phenomenon is observed for MBBI in the presence of Q8. Upon addition of one equivalent of Tyr-Leu-Ala to the Q8•MBBI complex (1:1:1 mixture, Figure $4 \mathrm{c}$ ), the chemical shifts of both MBBI signals return to those of free MBBI, whereas all signals corresponding to the Tyr and Leu residues move upfield, and those of the Ala residue move downfield. Based on these results, an in corroboration with the fluorescence data, we conclude that the peptide competitively displaces MBBI from the Q8 cavity. Moreover, it is clear that both Tyr and Leu side chains are bound within the cavity of Q8, and that the Ala side chain is located near the Q8 portal. In the absence of MBBI, the 1:1 mixture of Tyr-Leu-Ala and Q8 (Figure $4 \mathrm{~d}$ ) shows identical peptide signals as the 1:1:1 mixture of Tyr-Leu-Ala, MBBI, and Q8. This result shows that MBBI does not influence the binding mode of Tyr-Leu-Ala with Q8. The 1:2 mixture of Q8 and Tyr-Leu-Ala (Figure 4 e) has a spectrum that is identical to that of the Q8 $\bullet$ Tyr-Leu-Ala complex in addition to a second set of resonances corresponding to free peptide (Figure $4 \mathrm{f}$ ). These spectra confirm that Tyr-Leu-Ala binds to Q8 in a 1:1 stoichiometry and provides evidence that the kinetics of exchange between bound and unbound states is slow on the NMR timescale, thus suggesting a slow dissociation rate and a high binding affinity. The presence of the 1:1 peptide:Q8 complex was confirmed by ESI-MS (Figure S2).

The slow exchange kinetics observed for the Q8 $\bullet$ Tyr-Leu-Ala complex facilitated the acquisition of a ROESY spectrum (Figure S24) in order to gain more information on the conformation of the peptide in the complex. Nine NOEs were observed between the side chains of Tyr and Leu residues (interresidue, see Table S1), and seventeen NOEs were observed between the peptide and Q8 (intermolecular). On the basis of these data, a semiempirical computational model was constructed (Figure 5) that is consistent with the NMR data. The model shows the side chains of Tyr and Leu residues bound snugly within the Q8 cavity. It also suggests electrostatic stabilization of the complex via five putative hydrogen bonds between peptide $\mathrm{NH}$ and $\mathrm{NH}_{3}{ }^{+}$groups and Q8 carbonyl oxygens. 


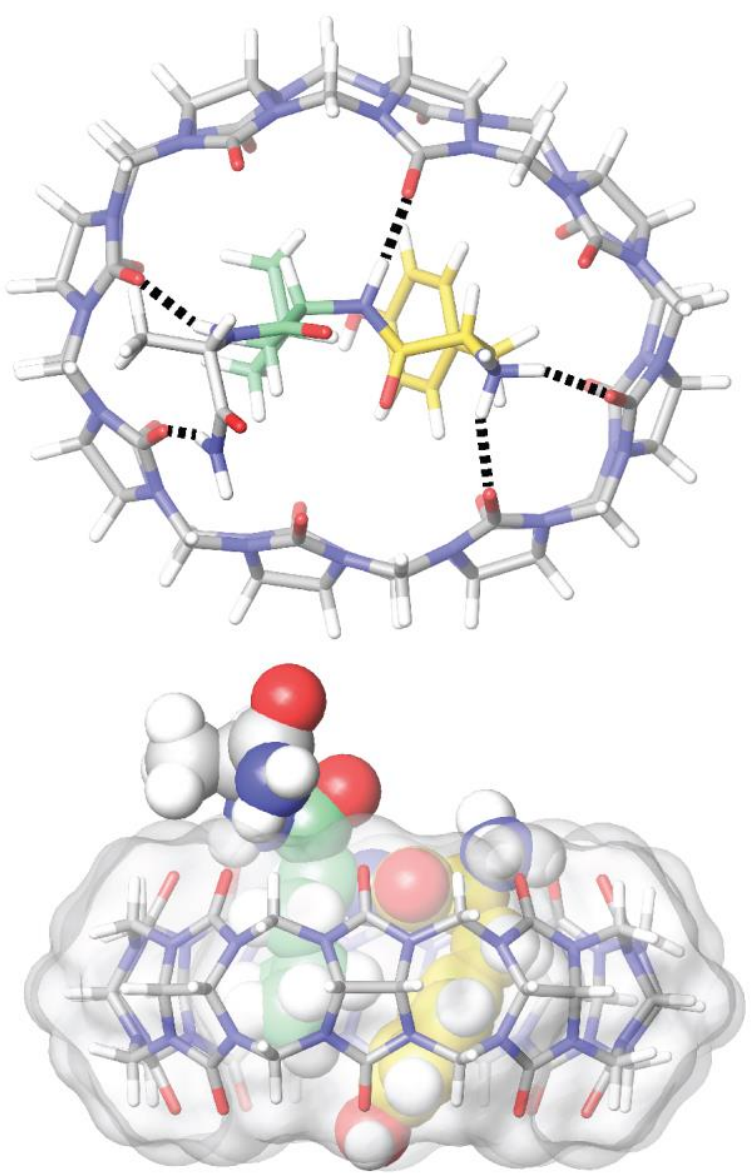

Figure 5. Semiempirical model of the Q8•Tyr-Leu-Ala complex. The model was built in Maestro by manually docking the peptide to fit the side chains of Tyr and Leu residues inside the Q8 cavity, and then subjecting the model to a geometry minimization in the OPLS 2005 molecular mechanics force field with implicit solvent using upper-bound distance restraints generated from the nine inter-residue (Tyr-to-Leu) NOEs observed in the ROESY spectrum (Figure S24).

In contrast to Tyr-Leu-Ala, studies of the sequence isomer Tyr-Ala-Leu reveal substantially different results. In the ${ }^{1} \mathrm{H}$ NMR spectrum of Tyr-Ala-Leu combined with Q8 and MBBI (Figure S28), the MBBI peaks broaden, but their chemical shifts do not return to those of free MBBI. The Tyr peaks shift upfield and broaden substantially, and the Ala and Leu peaks broaden slightly, but their chemical shifts do not change appreciably. These observations corroborate with the fluorescence data to demonstrate that MBBI and Tyr are bound simultaneously within the Q8 cavity. When Tyr-Ala-Leu is combined with Q8 (Figure S26), the Tyr peaks perturb upfield significantly, and they broaden but not as much as when MBBI is present. The Ala and Leu peaks broaden slightly, and their chemical shifts do not perturb significantly. In the 2:1 Tyr-Ala-Leu:Q8 mixture (Figure S27), the peaks observed in the 1:1 mixture broaden much further, and no new set of peaks was observed. These results suggest that chemical exchange kinetics are intermediate on the NMR time scale and are consistent with low-affinity binding. Unfortunately, the lack of spectral resolution limits the depth of the NMR analysis. It is clear, however, that the peaks corresponding only to the side chain of Tyr experience a significant upfield perturbation in chemical shift in the presence of Q8, and thus we conclude that only Tyr binds within the Q8 cavity. The 1:1 Q8•Tyr-Ala-Leu complex was confirmed by ESI-MS (Figure S4).

Extension of the Tyr-Var Motif. The contrasting results of Tyr-Leu-Ala and Tyr-Ala-Leu described above suggest that the Tyr and Leu residues need to be neighbors in order to bind in a 1:1 complex with Q8 and with the inclusion of both side chains. It was not clear, however, whether the Tyr- 
Leu binding site needs to be located at the N-terminus. To address this question, the sequence isomer Ala-Tyr-Leu was synthesized, purified, and characterized by ${ }^{1} \mathrm{H}$ NMR and ESI-MS (Figures S5 and S29). The NMR spectrum of Ala-Tyr-Leu in combination with Q8 (Figure S30) reveals the upfield perturbation of chemical shifts for Tyr and Leu side chains and thus their inclusion within the Q8 cavity. At substoichiometric ratios of Q8 to Ala-Tyr-Leu, two sets of peptide resonances are observed (free and bound), thus revealing a 1:1 complex and slow exchange on the NMR timescale (data not shown). The 1:1 Ala-Tyr-Leu $\bullet 8$ complex was observed by ESI-MS (Figure S6). Therefore, the Tyr-Leu binding site does not need to be located at the N-terminus.

Having established the unique nature of the Tyr-Leu binding site, we then wondered whether this motif could extend beyond Var = Leu. In the fluorescence screen described above, Var = Leu was chosen for further study because Tyr-Var 1 -Ala led to an increase in fluorescence, Tyr-Ala-Var 2 caused a decrease in fluorescence, and the difference between their changes in fluorescence intensity was the largest (23\%). Among the other peptides for which this pattern was observed, large differences were also observed for Var $=$ Phe $(21 \%)$ and Tyr $(18 \%)$. Therefore, the peptides Tyr-Phe-Ala, Tyr-Ala-Phe, Tyr-Tyr-Ala, and Tyr-Ala-Tyr were individually synthesized, purified, and characterized by ${ }^{1} \mathrm{H}$ NMR and ESI-MS (Figures S9, S11, S13, S15, S37, S39, S41, and S43) in order to probe the possible extension of this binding motif. The ${ }^{1} \mathrm{H}$ NMR spectra of Tyr-Phe-Ala and Tyr-Tyr-Ala show upfield perturbation of both aromatic side chains upon binding to Q8, indicating simultaneous inclusion within the host (Figures S38 and 42). In addition, the 2:1 mixtures of peptide:Q8 show a set of bound peptide resonances and a set of unbound peptide resonances (data not shown), and thus the kinetics of exchange is slow on the NMR timescale, and the complexes had a peptide:Q8 stoichiometry of 1:1. In both cases, a 1:1 peptide:Q8 stoichiometry was corroborated by ESI-MS (Figures S10 and S14). Similar to TyrAla-Leu, the ${ }^{1} \mathrm{H}$ NMR spectra of Tyr-Ala-Tyr and Tyr-Ala-Phe also show extensive signal broadening in the presence of Q8, thus complicating further analysis (Figures S40 and S44). On the basis of these data, we conclude that the Tyr-Var motif extends beyond Var = Leu to include Var $=$ Phe and Tyr.

The Tyr-Var•Q8 complex described here represents a novel binding motif in which the host accommodates the inclusion of two neighboring amino acid residues within its cavity and binds in a 1:1 stoichiometry. Due to the tight fit, the peptide has a very limited range of possible conformations, and thus Q8 serves as a template for the induced folding of the peptide. Although the inclusion of two amino acid residues within the cavity of a synthetic receptor and the induced folding of a peptide by a synthetic receptor are not entirely unique in the literature, they are rare, especially in aqueous media. In particular, work by Fujita and coworkers have demonstrated the sequence-specific recognition of multiple amino acids and the folding of peptides into alpha helix and beta-turn conformations by self-assembled coordination cages, with several complexes of $\mu \mathrm{M}$ affinity in aqueous solution. ${ }^{8,30-32}$

Thermodynamic Characterization of Peptide Binding. Thermodynamic constants for binding of peptides to Q8 were determined by isothermal titration calorimetry (ITC) (Table 1 and Figures S45S50). As expected, all complexes were observed to bind in a 1:1 peptide:Q8 ratio (" $n$ " values were within $10 \%$ of 1.0). We were surprised to observe that Tyr-Leu-Ala binds Q8 with an equilibrium dissociation constant $\left(\mathrm{K}_{\mathrm{d}}\right)$ value of $7.2 \mathrm{nM} .{ }^{33}$ This is, to the best of our knowledge, the strongest reported affinity of a synthetic receptor for an unmodified peptide in aqueous solution. This result is 
further highlighted by the fact that the buffer contains $10 \mathrm{mM}$ sodium, which binds to the host with a $\mathrm{K}_{d}$ value of $1 \mathrm{mM}^{10}$ and thus diminishes the

Table 1. Thermodynamic Data for Binding to Cucurbit[8]uril.

\begin{tabular}{lcccc}
\hline Peptide & \multicolumn{1}{c}{$\begin{array}{c}\mathrm{K}_{\mathrm{d}}^{\mathrm{a}} \\
(\mu \mathrm{M})\end{array}$} & $\begin{array}{c}\Delta \mathrm{G}^{\mathrm{b}} \\
(\mathrm{kcal} / \mathrm{mol})\end{array}$ & $\begin{array}{c}\Delta \mathrm{H}^{\mathrm{a}} \\
(\mathrm{kcal} / \mathrm{mol})\end{array}$ & $\begin{array}{c}-\mathrm{T} \Delta \mathrm{S}^{\mathrm{c}} \\
(\mathrm{kcal} / \mathrm{mol})\end{array}$ \\
\hline Tyr-Leu-Ala & $0.0072( \pm 0.0003)$ & $-11.2( \pm 0.1)$ & $-15.5( \pm 0.4)$ & $4.3( \pm 0.2)$ \\
Tyr-Ala-Leu & $34 .( \pm 7)$. & $-6.2( \pm 0.2)$ & $-9.5( \pm 0.1)$ & $3.4( \pm 0.1)$ \\
Ala-Tyr-Leu & $3.1( \pm 0.5)$ & $-7.6( \pm 0.2)$ & $-14.1( \pm 0.5)$ & $6.5( \pm 0.1)$ \\
Tyr-Lys-Ala & $0.20( \pm 0.03)$ & $-9.2( \pm 0.2)$ & $-15.5( \pm 0.1)$ & $6.3( \pm 0.2)$ \\
Tyr-Tyr-Ala & $0.70( \pm 0.06)$ & $-8.5( \pm 0.1)$ & $-16.1( \pm 0.2)$ & $7.7( \pm 0.2)$ \\
Tyr-Phe-Ala & $0.29( \pm 0.04)$ & $-9.0( \pm 0.2)$ & $-16.1( \pm 0.2)$ & $7.2( \pm 0.1)$ \\
\hline
\end{tabular}

a Mean values measured from at least two ITC experiments at $27{ }^{\circ} \mathrm{C}$ in $10 \mathrm{mM}$ sodium phosphate, $\mathrm{pH}$ 7.0. ${ }^{\mathrm{b}}$ Gibbs free energy values calculated from $\mathrm{K}_{\mathrm{d}}$ values. Standard deviations for $\Delta \mathrm{G}$ values were calculated as the relative error observed in $K_{d}$, due to their relationship by a natural logarithm. ${ }^{\mathrm{c}}$ Entropic contributions to $\Delta \mathrm{G}$ calculated from $\mathrm{K}_{\mathrm{d}}$ and $\Delta \mathrm{H}$ values, with error propagated from that of $\mathrm{K}_{\mathrm{d}}$ and $\Delta \mathrm{H}$.

observed binding affinity by 10 -fold; therefore, we estimate the $\mathrm{K}_{\mathrm{d}}$ of the Tyr-Leu-Ala•Q8 complex to be subnanomolar in pure water. The next highest affinity reported in the literature is the binding of PheGly by Q7 in pure water $\left(\mathrm{K}_{\mathrm{d}}=33 \mathrm{nM}\right)$, reported by Kim, Inoue, and coworkers. ${ }^{34}$ The strong affinity of Tyr-Leu-Ala is consistent with the slow exchange kinetics observed in NMR data. The peptide Ala-TyrLeu showed a 430-fold loss in affinity compared to Tyr-Leu-Ala. Therefore, the Tyr-Leu sequence can be bound by Q8 at both $\mathrm{N}$-terminal and non-N-terminal positions, but there is substantial specificity for N-terminal Tyr-Leu.

The sequence isomer Tyr-Ala-Leu binds Q8 with a $\mathrm{K}_{d}$ value of $34 \mu \mathrm{M}$, which is 4700 -fold weaker than its sequence isomer Tyr-Leu-Ala. This is exceptionally high sequence-specificity for a synthetic receptor in aqueous solution, second only to the study reported by Kim, Inoue, and coworkers for the binding of Phe-Gly vs. Gly-Phe by Q7 (23,000-fold) ${ }^{34}$ In their study and others, ${ }^{28,35}$ specificity for the N-terminal Phe is thought derive from the location of the aromatic residue relative to the charged groups at each terminus, whereas in the current study, the specificity of Tyr-Leu-Ala vs. Tyr-Ala-Leu is thought to derive from inclusion of the second side chain within the Q8 cavity. This hypothesis is supported by the enthalpy and entropy data (Table 1). The binding of both Tyr-Leu-Ala and Tyr-Ala-Leu is enthalpically driven and entropically unfavorable. The $5.0 \mathrm{kcal} / \mathrm{mol}$ difference in the free energy of complexation is due mostly to enthalpy $(6.0 \mathrm{kcal} / \mathrm{mol})$, whereas the entropic contribution to binding energy is similar to within $1 \mathrm{kcal} / \mathrm{mol}$. This observation suggests that, compared to the Tyr-Ala-Leu $\bullet \mathrm{Q} 8$ complex, the Tyr-Leu-Ala•Q8 complex is stabilized by the additional van der Waals interactions of the Leu side chain with the Tyr side chain and the walls of the Q8 cavity. Desolvation of the isobutyl group may contribute to the entropic stabilization but would be offset by the immobilization of the side chain within the tightly packed cavity. The low affinity of the Tyr-Ala-Leu complex was shared by the other two Tyr-Ala-Var2 peptides (Tyr-Ala-Tyr and Tyr-Ala-Phe) whose ITC data proved difficult to fit (data not shown). 
The $\mathrm{K}_{\mathrm{d}}$ values for Tyr-Phe-Ala and Tyr-Tyr-Ala were 0.29 and $0.70 \mu \mathrm{M}$, respectively. These submicromolar affinities are strong but significantly weaker than that of Tyr-Leu-Ala. Looking back at the fluorescence data from the initial library screen, Tyr-Phe-Ala (14\%) and Tyr-Tyr-Ala (13\%) showed a lower extent of fluorescence enhancement than Tyr-Leu-Ala (19\%) upon binding to Q8. This observation is consistent with a model in which the extent of fluorescence enhancement is proportional to the affinity of the peptide and thus the extent of MBBI displacement at a given concentration. Those data show $\operatorname{Var}_{1}=$ Lys (i.e., Tyr-Lys-Ala) as the peptide that induces the greatest extent of fluorescence enhancement upon binding to Q8 (21\%), and so we synthesized, purified, and characterized Tyr-LysAla by ${ }^{1} \mathrm{H}$ NMR and ESI-MS (Figures S7 and S31). As expected, Tyr-Lys-Ala interacted with Q8 in a similar manner the other Tyr-Var peptides by ${ }^{1} \mathrm{H}$ NMR and ESI-MS (Figures S8 and S32-S34). A semiempirical model (Figure S37) based on ROESY data (Figure S36) is consistent with the inclusion of both side chains, with additional interaction of the side chain ammonium group with the opposite carbonyl portal of Q8. Tyr-Lys-Ala binds Q8 with a $\mathrm{K}_{\mathrm{d}}$ value of $0.20 \mu \mathrm{M}$, which is stronger than that of Tyr-Tyr-Ala and Tyr-Phe-Ala but weaker than that of Tyr-Leu-Ala. Although we expected Tyr-Lys-Ala to bind a little stronger than Tyr-Leu-Ala, based on the fluorescence screen, the observed discrepancy is within the error of the fluorescence screen.

Phenylalanine-Terminated Peptides. The library screen for phenylalanine-terminated peptides (Figure S51) showed that the fluorescence intensity of the Q8•MBBI complex was consistently enhanced upon adding peptide. We thought (vide supra) this result was due to the binding of two equivalents of peptide to Q8, because we had observed this result previously for Phe-Gly-Gly. ${ }^{28}$ In light of the results reported here on tyrosine-terminated peptides, however, we revised our thinking on how Phe-terminated peptides may displace MBBI. Given the similarity in structure between Phe and Tyr, we predicted that Phe-Leu-Ala, for example, should bind Q8 in a similar fashion as Tyr-Leu-Ala.

To test this prediction, we synthesized and characterized Phe-Leu-Ala and Phe-Ala-Leu and studied their interaction with Q8 by ITC, NMR, and ESI-MS (Figures S54-S68). Prior work has shown Pheterminated peptides to bind with higher affinity than Tyr-terminated peptides to Qn hosts, ${ }^{6}$ presumably due to the greater hydrophobicity of Phe. The semiempirical model of the Q8•Tyr-Leu-Ala complex (Figure 5) shows the tyrosine hydroxyl group making no direct contact with peptide or Q8, and thus it was reasonable to predict even stronger binding for Phe-Leu-Ala. The ITC experiments for binding of Phe-Leu-Ala to Q8 reveal a 1:1 Q8:peptide stoichiometry and an average $\mathrm{K}_{\mathrm{d}}$ value of $0.43( \pm 0.12) \mu \mathrm{M}$, which is significantly weaker than that of the Tyr analogue. This is consistent with our prediction that Phe-Leu-Ala should bind Q8 in a similar fashion as Tyr-Leu-Ala, but it was surprising that the affinity is weaker. The ESI-MS data confirm the presence of 1:1 and 2:1 peptide:Q8 complexes. The NMR data confirm the inclusion of Phe and Leu sidechains within the Q8 cavity and thus a similar mode of binding as Tyr-Leu-Ala.

In contrast to the results for Tyr-containing peptides, however, the ITC, NMR, and ESI-MS data for the sequence isomer Phe-Ala-Leu reveal a 2:1 peptide:Q8 complex as the dominant species. ITC experiments show positive cooperativity, as observed for Phe-Gly-Gly. ${ }^{28}$ NMR experiments show the 2:1 Phe-Ala-Leu:Q8 complex, even at substoichiometric ratios (data not shown), and the inclusion of only the Phe sidechain within the Q8 cavity, which is also consistent with prior work on Phe-Gly-Gly. 
These results demonstrate that the increase in fluorescence intensity observed in the library screen can be due to 1:1 or 2:1 peptide:Q8 binding, depending on the peptide sequence.

\section{CONCLUSIONS}

This paper explores the effects of sequence context on the binding of the synthetic receptor cucurbit[8]uril (Q8) to peptides containing an N-terminal aromatic residue. Using MBBI as a fluorescent indicator, we found that peptides containing an N-terminal Trp bind to Q8 as a second guest, causing a decrease in MBBI fluorescence, whereas Phe-terminated peptides displace MBBI from the Q8 cavity, causing an increase in fluorescence. This novel use of a fluorescent indicator as both turn-off and turn-on sensor based on two different binding modes led to the discovery that the mode of binding for Tyr-terminated peptides depends on the amino acid sequence. Further study by ${ }^{1} \mathrm{H}$ NMR spectroscopy and ESI-MS showed that four peptides of sequence Tyr-Var-Ala (Var = Leu, Lys, Phe, and Tyr), as well as Phe-Leu-Ala, displace MBBI and bind to Q8 in a 1:1 stoichiometry with the side chains of both the terminal residue and its immediate neighbor bound inside the Q8 cavity. Isothermal titration calorimetry experiments corroborated the stoichiometry and gave submicromolar affinity $\mathrm{K}_{\mathrm{d}}$ values for these peptides. Remarkably, Tyr-Leu-Ala bound to Q8 with a $\mathrm{K}_{d}$ value of $7.2 \mathrm{nM}$ in $10 \mathrm{mM}$ sodium phosphate buffer, which is, to the best of our knowledge, the highest reported affinity for a synthetic receptor binding to an unmodified peptide in aqueous solution. Moving the Leu residue further away (e.g., TyrAla-Leu) markedly diminished binding affinity (4300-fold), showing exceptionally high sequencespecificity. In the Tyr-Ala-Leu•Q8 complex, only the Tyr residue bound within the Q8 cavity, allowing a second equivalent of peptide or MBBI to bind simultaneously. This result is important because it brings us significantly closer to the long-standing goal of developing a synthetic receptor that can bind peptides in aqueous media with sufficiently high affinity and specificity as to make them, like antibodies, practical for applications in biochemistry and biotechnology. Q8 binding to Tyr-Leu-Ala is not a general approach to peptide recognition, but Q8 has the advantages of being small, highly stable, recyclable, ${ }^{36}$ and many orders less expensive than a monoclonal antibody. Moreover, this peptide sequence is minimal and can be readily engineered into recombinant proteins for use as an affinity tag for biological applications. ${ }^{37-39}$

\section{ASSOCIATED CONTENT}

\section{Supporting Information}

Experimental details and mass spectrometry, ${ }^{1} \mathrm{H}$ NMR, isothermal titration calorimetry, and fluorescence data. This material is available free of charge via the Internet at http://pubs.acs.org.

\section{AUTHOR INFORMATION}

\section{Corresponding Author}

* to whom correspondence should be addressed: aurbach@trinity.edu

\section{Author Contributions}

The manuscript was written through contributions of all authors. All authors have given approval to the final version of the manuscript.

\section{Funding Sources}


This work was supported in part by grants from the Welch Foundation (W-1640 and W-0031), the National Science Foundation (CHE-1309978, CHE-0748483, and CHE-0957839), the Henry Dreyfus Teacher-Scholar Awards Program, and Trinity University. Omar Ali was a Beckman Scholar.

\section{ACKNOWLEDGMENT}

We gratefully acknowledge Prof. Christopher Bielawski for providing the MBBI used in this study. This work was supported in part by grants from the Welch Foundation (W-1640 and W-0031), the National Science Foundation (CHE-1309978, CHE-0748483, and CHE-0957839), the Henry Dreyfus Teacher-Scholar Awards Program, and Trinity University. Omar Ali was a Beckman Scholar.

\section{REFERENCES}

E2656.

(1) Peng, H.-P.; Lee, K. H.; Jian, J.-W.; Yang, A.-S. Proc. Natl. Acad. Sci. USA 2014, 111,

(2) Peczuh, M. W.; Hamilton, A. D. Chem. Rev. 2000, 100, 2479.

(3) Yin, H.; Hamilton, A. D. Angew. Chem. Int. Ed. 2005, 44, 4130.

(4) Houk, K. N.; Leach, A. G.; Kim, S. P.; Zhang, X. Angew. Chem. Int. Ed. 2003, 42, 4872.

(5) Schmuck, C. Synlett 2011, 1798.

(6) Urbach, A. R.; Ramalingam, V. Israel J. of Chem. 2011, 51, 664.

(7) Wright, A. T.; Griffin, M. J.; Zhong, Z. L.; McCleskey, S. C.; Anslyn, E. V.; McDevitt, J. T. Angew. Chem. Int. Ed. 2005, 44, 6375.

(8) Tashiro, S.; Tominaga, M.; Kawano, M.; Therrien, B.; Ozeki, T.; Fujita, M. J. Am. Chem. Soc. 2005, 127, 4546.

(9) Wehner, M.; Janssen, D.; Shafer, G.; Schrader, T. Eur. J. Org. Chem. 2006, 2006, 138. 44,4844 .

(10) Lagona, L.; Mukhopadhyay, P.; Chakrabarti, S.; Isaacs, L. Angew. Chem. Int. Ed. 2005,

(11) Liu, S.; Ruspic, C.; Mukhopadhyay, P.; Chakrabarti, S.; Zavalij, P. Y.; Isaacs, L. J. Am. Chem. Soc. 2005, 127, 15959.

(12) Rekharsky, M. V.; Mori, T.; Yang, C.; Young, H. K.; Selvapalam, N.; Kim, H.; Sobransingh, D.; Kaifer, A. E.; Liu, S.; Isaacs, L.; Chen, W.; Moghaddam, S.; Gilson, M. K.; Kim, O.; Inoue, Y. Proc. Natl. Acad. Sci. USA 2007, 104, 20737.

(13) Cao, L.; Sekotur, M.; Zavalij, P. Y.; Mlinaric-Majerski, M.; Glaser, R.; Isaacs, L. Angew. Chem. Int. Ed. 2014, 53, 988.

(14) Lee, J. W.; Samal, S.; Selvapalam, N.; Kim, H. J.; Kim, K. Acc. Chem. Res. 2003, 36, 621.

(15) Liu, S. M.; Zavalij, P. Y.; Isaacs, L. J. Am. Chem. Soc. 2005, 127, 16798.

(16) Lagona, L.; Fettinger, J.; Isaacs, L. J. Org. Chem. 2005, 70, 10381.

(17) Isaacs, L.; Park, S.-K.; Liu, S. M.; Ko, Y. H.; Selvapalam, N.; Kim, Y.; Kim, H.; Zavalij, P. Y.; Kim, G.-H.; Lee, H.-S.; Kim, K. J. Am. Chem. Soc. 2005, 127, 18000.

(18) Huang, W. H.; Liu, S. M.; Zavalij, P. Y.; Isaacs, L. J. Am. Chem. Soc. 2006, 128, 14744.

(19) Kim, K.; Selvapalam, N.; Ko, Y. H.; Park, K. M.; Kim, D.; Kim, J. Chem Soc Rev 2007, 36,

267.

(20) Vinciguerra, B.; Cao, L.; Cannon, J. R.; Zavalij, P. Y.; Fenselau, C.; Isaacs, L. J. Am. Chem. Soc. 2012, 134, 13133.

(21) Lucas, D.; Isaacs, L. Org. Lett. 2011, 13, 4112.

(22) Nau, W. M.; Scherman, O. A. Israel J. Chem. 2011, 51, 492.

(23) Kim, J.; Jung, I.-S.; Kim, S.-Y.; Lee, E.; Kang, J.-K.; Sakamoto, S.; Yamaguchi, K.; Kim, K. J. Am. Chem. Soc. 2000, 122, 540.

(24) Kim, H.-J.; Heo, J.; Jeon, W. S.; Lee, E.; Kim, J.; Sakamoto, S.; Yamaguchi, K.; Kim, K. Angew. Chem. Int. Ed. 2001, 40, 1526.

(25) Bush, M. E.; Bouley, N. D.; Urbach, A. R. J. Am. Chem. Soc. 2005, 127, 14511.

(26) Ali, O. A.; Olson, E. M.; Urbach, A. R. Supramol. Chem. 2013, 25, 863. 
(27) Biedermann, F.; Rauwald, U.; Cziferszky, M.; Williams, K. A.; Gann, L. D.; Guo, B. Y.; Urbach, A. R.; Bielawski, C. W.; Scherman, O. A. Chem. Eur. J. 2010, 16, 13716. 12574.

(28) Heitmann, L. M.; Taylor, A. B.; Hart, P. J.; Urbach, A. R. J. Am. Chem. Soc. 2006, 128,

(29) Moon, K.; Kaifer, A. E. Org. Lett. 2004, 6, 185.

(30) Tashiro, S.; Kobayashi, M.; Fujita, M. J. Am. Chem. Soc. 2006, 128, 9280.

(31) Dolain, C.; Hatakeyama, Y.; Sawada, T.; Tashiro, S.; Fujita, M. J. Am. Chem. Soc. 2010, 132,5564 .

(32) Hatakeyama, Y.; Sawada, T.; Kawano, M.; Fujita, M. Angew. Chem. Int. Ed. 2009, 48, 8695.

(33) This value was obtained by titration in the presence of 100 -fold excess MBBI as a weak competitor (Figure S45).

(34) Rekharsky, M. V.; Yamamura, H.; Ko, Y. H.; Selvapalam, N.; Kim, K.; Inoue, Y. Chem. Comm. 2008, 2236.

(35) Chinai, J. M.; Taylor, A. B.; Ryno, L. M.; Hargreaves, N. D.; Morris, C. A.; Hart, P. J.; Urbach, A. R. J. Am. Chem. Soc. 2011, 133, 8810.

8587.

(36) Ramalingam, V.; Kwee, S. K.; Ryno, L. M.; Urbach, A. R. Org. Biomol. Chem. 2012, 10,

(37) Nguyen, H. D.; Dang, D. T.; van Dongen, J. L. J.; Brunsveld, L. Angew. Chem. Int. Ed. 2010, 49, 895.

2915.

(38) Dang, D. T.; Nguyen, H. D.; Merkx, M.; Brunsveld, L. Angew. Chem. Int. Ed. 2013, 52,

(39) Gonzalez-Campo, A.; Brasch, M.; Uhlenheuer, D. A.; Gomez-Casado, A.; Yang, L.; Brunsveld, L.; Huskens, J.; Jonkheijm, P. Langmuir 2012, 28, 16364. 


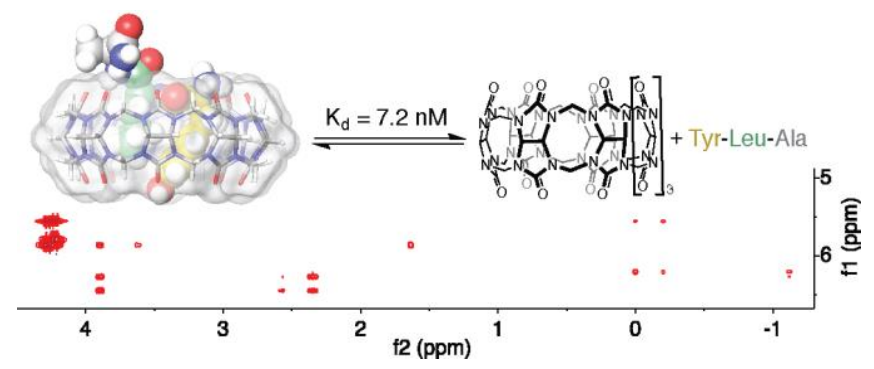

